\title{
Treadmill Exercise Ameliorates Chemotherapy-Induced Muscle Weakness and Central Fatigue by Enhancing Mitochondrial Function and Inhibiting Apoptosis
}

\author{
Sang-Seo Park ${ }^{1}$, Hye-Sang Park ${ }^{1}$, Hyungmo Jeong ${ }^{2}$, Hyo-Bum Kwak ${ }^{3}$, Mi-Hyun No ${ }^{3}$ Jun-Won Heo ${ }^{3}$, Su-Zi Yoo ${ }^{3}$, Tae-Woon Kim ${ }^{1,4}$ \\ ${ }^{1}$ Department of Physiology, College of Medicine, Kyung Hee University, Seoul, Korea \\ ${ }^{2}$ Department of Anesthesiology and Pain Medicine, Graduate School, Kyung Hee University, Seoul, Korea \\ ${ }^{3}$ Department of Kinesiology, Inha University, Incheon, Korea \\ ${ }^{4}$ Exercise Rehabilitation Research Institute, Department of Exercise \& Health Science, Sangmyung University, Seoul, Korea
}

Purpose: Chemotherapy is associated with the side effects including damage to the mitochondrial DNA. Doxorubicin (DOX) serves as a chemotherapeutic agent for the patients with breast cancer or prostate cancer. DOX causes muscle weakness and fatigue. We investigated the effects of treadmill exercise on DOX-induced apoptosis and mitochondrial dysfunction in relation to central fatigue. For this study, we used the rat model of DOX-induced muscle damage.

Methods: DOX ( $2 \mathrm{mg} / \mathrm{kg}$ ) was intraperitoneally injected 1 time per week for 4 weeks. Treadmill running continued 5 days per week for 4 weeks. Muscle strength and fatigue index in the gastrocnemius were measured. Immunohistochemistry for the expressions of tryptophan hydroxylase (TPH) and 5-hydroxytryptamine (5-HT) in the dorsal raphe was conducted. We used western blot analysis for the expressions of $\mathrm{Bax}, \mathrm{Bcl}-2$, and caspases-3 in the gastrocnemius. Mitochondrial function in the gastrocnemius was also evaluated.

Results: DOX treatment decreased muscle strength with increase of fatigue index in the gastrocnemius. Mitochondria function was deteriorated and apoptosis in the gastrocnemius was enhanced by DOX treatment. Expressions of TPH and 5-HT in the dorsal raphe were increased by DOX treatment. Treadmill exercise attenuated DOX-induced muscle fatigue and impairment of mitochondria function. Apoptosis in the gastrocnemius was inhibited and over-expression of TPH and 5-HT was suppressed by treadmill exercise.

Conclusions: Apoptosis was enhanced and mitochondria function was deteriorated by DOX treatment, resulting in muscle weakness and central fatigue. Treadmill exercise suppressed apoptosis and prevented deterioration of mitochondria function in muscle, resulting in alleviation of muscle weakness and central fatigue during DOX therapy.

Keywords: Chemotherapy; Doxorubicin; Muscle strength; Fatigue; Mitochondrial function; Apoptosis

- Fund/Grant Support: This work was supported by the Ministry of Education of the Republic of Korea and the National Research Foundation of Korea (NRF-2017S1A5B5A07060388).

- Research Ethics: All animal experimental procedures conformed to the regulations stipulated by the National Institutes of Health (NIH) and the guidelines of the Korean Academy of Medical Science. This study was approved by the Kyung Hee university Institutional Animal Care and Use Committee (Seoul, Korea) (KHUASP [SE]-15-086).

- Conflict of Interest: No potential conflict of interest relevant to this article was reported.

Corresponding author: Tae-Woon Kim (iD http://orcid.org/0000-0001-8832-0874 Exercise Rehabilitation Research Institute, Department of Exercise \& Health Science, Sangmyung University, 20, Hongjimun 2-gil, Jongno-gu, Seoul 03016, Korea E-mail: twkim0806@naver.com / Tel:+82-2-961-0282 / Fax:+82-2-964-2195 Submitted: January 2, 2019 / Accepted after revision: February 16, 2019
This is an Open Access article distributed under the terms of the Creative Commons Attribution Non-Commercial License (http://creativecommons.org/licenses/by-nc/4.0/) which permits unrestricted non-commercial use, distribution, and reproduction in any medium, provided the original work is properly cited. 


\section{INTRODUCTION}

Chemotherapy is known to induce diverse side effects including damage to the mitochondrial DNA [1]. Doxorubicin (DOX) is a well-known anticancer drug for the treating of solid tumors and hematologic malignancies. DOX is a drug of choice for the patients with breast cancer or prostate cancer. However, DOX is known to cause muscle atrophy and fatigue during therapy $[2,3]$. Chemotherapy including DOX induces changes of mitochondrial morphology and function in heart, brain, and muscle [4-6]. Although DOX has been used to treat many cancers, its use is limited by its side effects [7,8].

Chemotherapy-induced apoptosis demonstrates diverse apoptosis-related biological candidates, such as increased DNA fragmentation in skeletal muscle [9], activation of several caspases (e.g., 3, 6, and 9) [10,11], cytochrome c release [12], and increased $\mathrm{Bax} / \mathrm{Bcl}-2$ ratio $[9,10,12]$. Mitochondrial DNA damage caused by reactive oxygen species (ROS) results in cellular dysfunction [13]. Chemotherapy enhances ROS production and increases intracellular $\mathrm{Ca}^{2+}$ concentration [14]. And then, these events are associated with the elevated levels of tumor necrosis factor-alpha (TNF- $\alpha$ ) and other inflammatory cytokines. The production of inflammatory cytokines, local inflammation, and oxidative stress leads to cellular injury, and eventually causes cell death $[15,16]$. DOX increases the production of inflammatory cytokines such as TNF- $\alpha$ and reduces muscle contraction $[15,17]$. This drug also increases free radicals in muscle, in turn, reduces antioxidant level, thereby leading to a loss in muscle mass $[18,19]$. Throughout the course of chemotherapy, DOX causes fatigue by inducing mitochondrial damage, oxidative stress, and apoptosis $[15,19,20]$.

Exercise regimen has been suggested for the maintaining physical fitness against chemotherapy. Exercise training exerts protective effect on chemotherapy-evoked myopathy in skeletal muscle [20]. Exercise reduces the loss of muscle function by increasing antioxidant enzyme level [17,21]. Continuous exercise improves mitochondrial function, which is impaired by chemotherapy [22].

Fatigue is classified as central fatigue (brain) or peripheral fatigue (muscle) based on the site of occurrence. Central fatigue is associated with impaired exercise performance due to change of neurotransmitters [23]. Serotonin is a neurotransmitter that regulates emotions, depression, lethargy, anxiety, and schizophrenia [24]. Serotonin has been suggested to be associated with the exercise-induced central fatigue [25]. In many studies, neurotransmitters, such as serotonin and dopamine, have been proposed as a causative chemical of physical activity-induced central fatigue [26,27]. In this study, we investigated the effects of treadmill exercise on DOX-induced apoptosis and mitochondrial dysfunction in relation to central fatigue. For this study, we used the rat model of DOX-induced muscle damage.

\section{MATERIALS AND METHODS}

\section{Experiment Animals}

The procedure of this study followed the guidelines of Korean Academy of Medical Science and the regulations of National Institutes of Health and. We obtained approval from the Kyung Hee University Institutional Animal Care and Use Committee (Seoul, Korea) (KHUASP [SE]-15-086). We divided the SpragueDawley male rats (8 weeks in age, $200 \mathrm{~g}$ in body weight) randomly into 4 groups: control group, control with exercise group, DOX-treated group, and DOX-treated with exercise group.

\section{DOX-Treated Muscle Damage Model}

We obtained DOX from Tokyo Chemical Industry (Tokyo, Japan). To induce the experimental model of muscle damage, DOX $(2 \mathrm{mg} / \mathrm{kg})$ dissolved in saline was intraperitoneally injected to the rats, 1 time per week for 4 weeks [28].

\section{Exercise Protocol}

The rats in the exercise groups began their running on a treadmill. The running intensity composed of warm up during 5 minutes at $3 \mathrm{~m} / \mathrm{min}$, main exercise during 30 minutes at $10 \mathrm{~m} /$ min and cool down during 5 minutes at $3 \mathrm{~m} / \mathrm{min}$ with a 0 inclination. The rats performed treadmill running one time per 1 day and 6 days per week, during 4 consecutive weeks.

\section{Grip Strength and Fatigue Index}

Using a grip strength meter (BIO-GS3, Bioseb, Vitrolles, France), muscle strength was determined. After the rat pulled the wire with the paw, we pulled the tail of the rat. The maximum grip strength was recorded as gram until the rat did not release the wire when gently pulling the rat's back. The test repeated 3 times in the one session, and the mean value was used as the data of maximal grip strength for each rat. We performed 6 sessions with one rat for measurement of muscle strength. The fatigue index was calculated according to the following formula. Fatigue index $=(1-[5$ th measurement of muscle strength +6 th measurement $] /[1$ st measurement +2 nd measurement $]) \times 100$. 


\section{Measurement of Mitochondrial $\mathrm{O}_{2}$ Respiration}

High resolution $\mathrm{O}_{2}$ consumption measurement was conducted by the Oroboros Oxygraph-2 K (Innsbruck, Austria) at $37^{\circ} \mathrm{C}$, according to the previous study [28]. After permeabilization and washing the gastrocnemius, it was moved to the chamber with $2 \mathrm{~mL}$ of MiR05 respiration buffer $\left(60 \mathrm{mM} \mathrm{C}{ }_{6} \mathrm{H}_{11} \mathrm{O}_{7} \mathrm{~K}\right.$, $130 \mathrm{mM}$ sucrose, $1 \mathrm{mM}$ ethyleneglycol tetra-acetic acid (EGTA), $10 \mathrm{mM} \mathrm{K}_{2} \mathrm{HPO}_{4}, 3 \mathrm{mM} \mathrm{MgCl}, 1 \mathrm{mg} / \mathrm{mL}$ BSA, and $20 \mathrm{mM}$ HEPES, pH 7.4). The protocol consisted of $2 \mathrm{mM}$ glutamate with $1 \mathrm{mM}$ malate (first), $2 \mathrm{mM}$ adenosine diphosphate infusion (ADP) (second), 3mM succinate (third). The completeness of the outer mitochondrial membrane was confirmed to be without increased respiration by addition of cytochrome $c$. We also confirmed that there is no endogenous substrate for permeabilized myotubes prior to protocol. When there is no added substrate, we washed muscle and measured ADP-stimulated respiration. The $\mathrm{O}_{2}$ consumption rate was presented as picomole/ $\mathrm{min} / \mathrm{mg}$ tissue weight.

\section{Measurement of Mitochondria $\mathrm{H}_{2} \mathrm{O}_{2}$ Emission}

According to the previous study [28], $\mathrm{H}_{2} \mathrm{O}_{2}$ emission was determined by $\Delta \mathrm{F} / \mathrm{min}$ at $37^{\circ} \mathrm{C}$ during state 4 respiration $(10-\mu \mathrm{g} / \mathrm{mL}$ oligomycin) through continually monitoring of the oxidation of Amplex Red (excitation/emission $\lambda=563 / 587 \mathrm{~nm}$ ). Mitochondrial $\mathrm{H}_{2} \mathrm{O}_{2}$ emission was measured by a Spex Fluormax 4 spectrofluorometer (HORIBA Jobin Yvon, Edison, NJ, USA) at $37^{\circ} \mathrm{C}$. The experiment was conducted with buffer $\mathrm{Z}$ added to Amplex Red, horseradish peroxidase, and oligomycin, an inhibitor of mitochondrial adenosine triphosphate synthase. The mitochondrial $\mathrm{H}_{2} \mathrm{O}_{2}$ emission protocol is as follows: $5 \mathrm{mM}$ glutamate and $2 \mathrm{mM}$ malate for complex I substrate, $10 \mathrm{mM}$ succinate for complex II substrate, and 10mM glycerol-3 phosphate for lipid substrate. The $\mathrm{H}_{2} \mathrm{O}_{2}$ emission rate was calculated after subtracting the background value from the reference value (standard curve) using the $\Delta \mathrm{F} / \mathrm{min}$ curve value. The result was presented as picomole $/ \mathrm{min} / \mathrm{mg}$ of tissue weight.

\section{Measurement of Mitochondria $\mathrm{Ca}^{2+}$ Retention Capacity}

To assess the openness of the permeability transition pore (PTP), mitochondrial $\mathrm{Ca}^{2+}$ retention capacity was determined, according to the previous study [28]. The mitochondrial $\mathrm{Ca}^{2+}$ retention capacity was analyzed using a Spex Fluormax 4 spectrofluorometer (HORIBA Jobin Yvon). By continuous monitoring of $\Delta \mathrm{F} / \mathrm{min}$ at $37^{\circ} \mathrm{C}$ during state 4 condition, overlaid traces of $\mathrm{Ca}^{2+}$-induced fluorescence changes by Calcium Green-5N were measured. After background $\Delta \mathrm{F}(1 \mu \mathrm{M}$ Calcium Green$5 \mathrm{~N}, 80 \mu \mathrm{M}$ EGTA, 0.5M glutamate, and 0.2M malate in Buffer $\mathrm{Z})$ was established, then we initiated the reaction by adding $\mathrm{Ca}^{2+}$ pulses $(30 \mu \mathrm{M})$ with wavelengths of excitation and emission at 506 and $532 \mathrm{~nm}$, respectively. Before the PTP opening (i.e., release of $\mathrm{Ca}^{2+}$ ), mitochondrial $\mathrm{Ca}^{2+}$ retention capacity was presented as picomole/mg of tissue weight.

\section{Tissue Preparation}

In order to sacrifice the rats, Zoletil 50 (10 mg/kg; Vibac Laboratories, Carros, France) was intraperitoneally injected into the rats for the anesthetizing. And then, 50mM phosphate-buffered saline (PBS) was transcardially infused. The rats were fixed using $4 \%$ paraformaldehyde in $100 \mathrm{mM}$ phosphate buffer $(\mathrm{pH}$, 7.4). After the brains were dissected, the brains were post-fixed during overnight and then treated by a $30 \%$ sucrose solution. We used a freezing microtome (Leica, Nussloch, Germany) to create sagittal sections of $40 \mu \mathrm{m}$ thick.

\section{Immunohistochemistry for Tryptophan Hydroxylase and 5-Hydroxytryptamine}

We performed immunohistochemistry to calculate the numbers of tryptophan hydroxylase (TPH)-positive and 5-hydroxytryptamine (5-HT)-positive cells in the dorsal raphe, according to the previous study [29]. The sections were treated with PBS for 10 minutes, after that, the sections were washed 3 times by PBS. And then, the sections were treated with $1 \% \mathrm{H}_{2} \mathrm{O}_{2}$ for 30 minutes, after that the sections were treated to mouse anti-TPH antibody (Oncogene Research Product, Cambridge, UK; 1:500) or to rabbit anti-5-HT antibody (Oncogene Research Product; 1:500) during overnight. The sections were then treated to biotinylated anti-mouse secondary antibody or to biotinylated anti-rabbit secondary antibody (Vector Laboratories, Burlingame, CA, USA) for 1 hour and 30 minutes. After incubation of the sections with avidin-biotin-peroxidase complex (Vector Laboratories) for 1 hour and 30 minutes, immunoreactivity was appeared by treating with a solution composing of $0.05 \% 3,3^{\prime}$-diaminobenzidine and $0.01 \% \mathrm{H}_{2} \mathrm{O}_{2}$ in $50 \mathrm{mM}$ Tris-buffer $(\mathrm{pH}$, 7.6) for 3 minutes. At room temperature, the slides were airdried during overnight, and Permount (Thermo Fisher Scientific Inc., Waltham, MA, USA) was used to mount.

\section{Western Blot Analysis for Bax, Bcl-2 and Caspases-3}

According to the previous study [30], we conducted western blotting. Tissue samples obtained by harvesting of gastrocne- 
mius muscle were lysed using protein lysis buffer. Colorimetric protein assay kit (Bio-Rad, Hercules, CA, USA) was used for the measuring of protein concentration. Protein $(20 \mu \mathrm{g})$ was separated on sodium dodecyl sulfate-polyacrylamide gels, then it was transferred to a nitrocellulose membrane (Schleicher \& Schuell GmbH, Dassel, Germany). For the primary antibodies, we used anti-mouse $\beta$-actin antibody (Santa Cruz Biotechnology, Santa Cruz, CA, USA; 1:1,000), anti-mouse Bax antibody (Santa Cruz Biotechnology; 1:1,000), anti-mouse Bcl-2 antibody (Santa Cruz Biotechnology; 1:1,000), and anti-rabbit caspase-3 antibody (Cell signaling Technology, Danvers, MA, USA; 1:1,000). For the secondary antibodies, we used horseradish peroxidase-conjugated anti-mouse antibodies (Santa Cruz Biotechnology; 1:3,000) for $\beta$-actin, Bax, Bcl-2, and anti-rabbit antibody (Santa Cruz Biotechnology; 1:5,000) for caspase-3. We calculate the detected bands using enhanced chemiluminescence detection system (Amersham Pharmacia Biotech GmbH, Freiburg, Germany).

\section{Statistical Analysis}

The results were statistically analyzed by IBM SPSS Statistics ver. 21.0 (IBM Co., Armonk, NY, USA). For the comparison among the groups, we used 1-way analysis of variance following Tukey post hoc test. Data are presented as the mean \pm standard error of the mean. Statistical significance was considered when P-value was less than 0.05 .

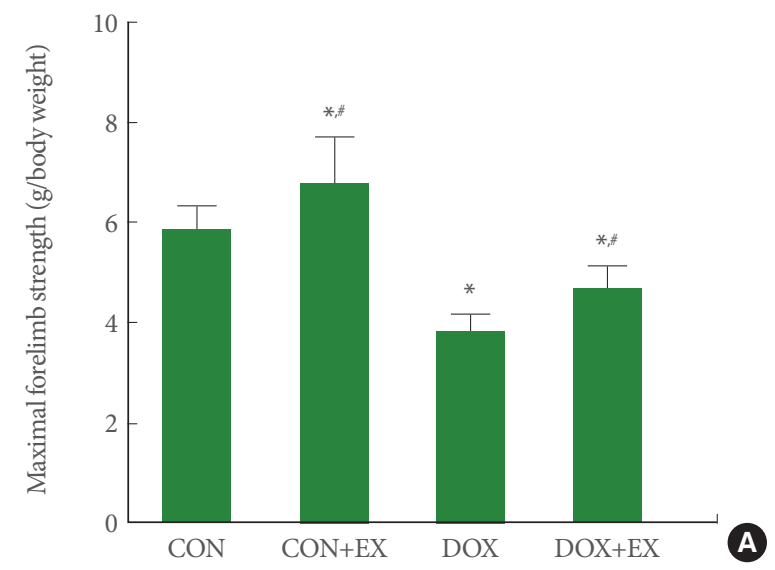

\section{RESULTS}

\section{Muscle Strength and Fatigue Index}

Fig. 1 shows the results of muscle strength and fatigue index. Muscle strength was decreased by DOX treatment $(\mathrm{P}<0.05)$, in contrast, treadmill exercise increased muscle strength in the DOX-treated group $(\mathrm{P}<0.05)$. Fatigue index was increased by DOX treatment $(\mathrm{P}<0.05)$, in contrast, treadmill exercise decreased fatigue index in the DOX-treated group $(\mathrm{P}<0.05)$.

\section{Expressions of TPH and 5-HT in Dorsal Raphe}

Fig. 2 shows the numbers of TPH-positive cells and 5-HT-positive cells in the dorsal raphe. Expression of TPH-positive cells in the dorsal raphe was increased by DOX treatment $(\mathrm{P}<0.05)$, in contrast, treadmill exercise decreased expression of TPHpositive cells in the DOX-treated group $(\mathrm{P}<0.05)$. Expression of 5-HT-positive cells in the dorsal raphe was increased by DOX treatment $(\mathrm{P}<0.05)$, in contrast, treadmill exercise decreased expression of 5-HT-positive cells in the DOX-treated group $(\mathrm{P}<0.05)$.

\section{Expressions of $\mathrm{Bax}, \mathrm{Bcl}-2$, and Caspases-3 in Gastrocnemius}

Fig. 3 shows the expressions of $\mathrm{Bax}, \mathrm{Bcl}-2$, and caspases- 3 in the gastrocnemius. Bax expression was increase and Bcl-2 expression was decreased by DOX treatment $(\mathrm{P}<0.05)$, in contrast, treadmill exercise decreased Bax expression and increased Bcl2 expression in the DOX-treated group $(\mathrm{P}<0.05)$. Caspases-3

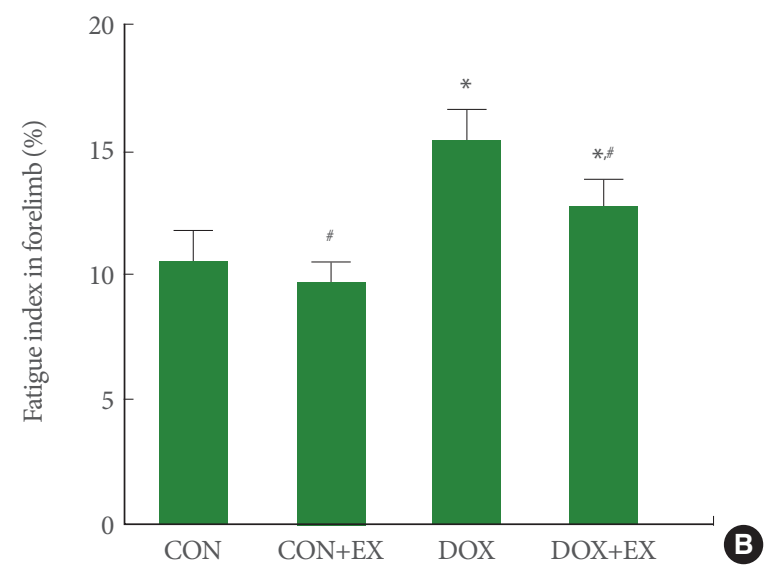

Fig. 1. The effect of treadmill exercise on muscle strength and fatigue index in gastrocnemius. (A) Muscle strength. (B) Fatigue index. $\mathrm{CON}$, control group; $\mathrm{CON}+\mathrm{EX}$, control with exercise group; DOX, doxorubicin (DOX)-treated group; DOX+EX, DOX-treated with exercise group. Data are expressed as the mean \pm standard error of the mean. ${ }^{*} \mathrm{P}<0.05$ compared to control group. ${ }^{*} \mathrm{P}<0.05$ compared to DOX-treated group. 

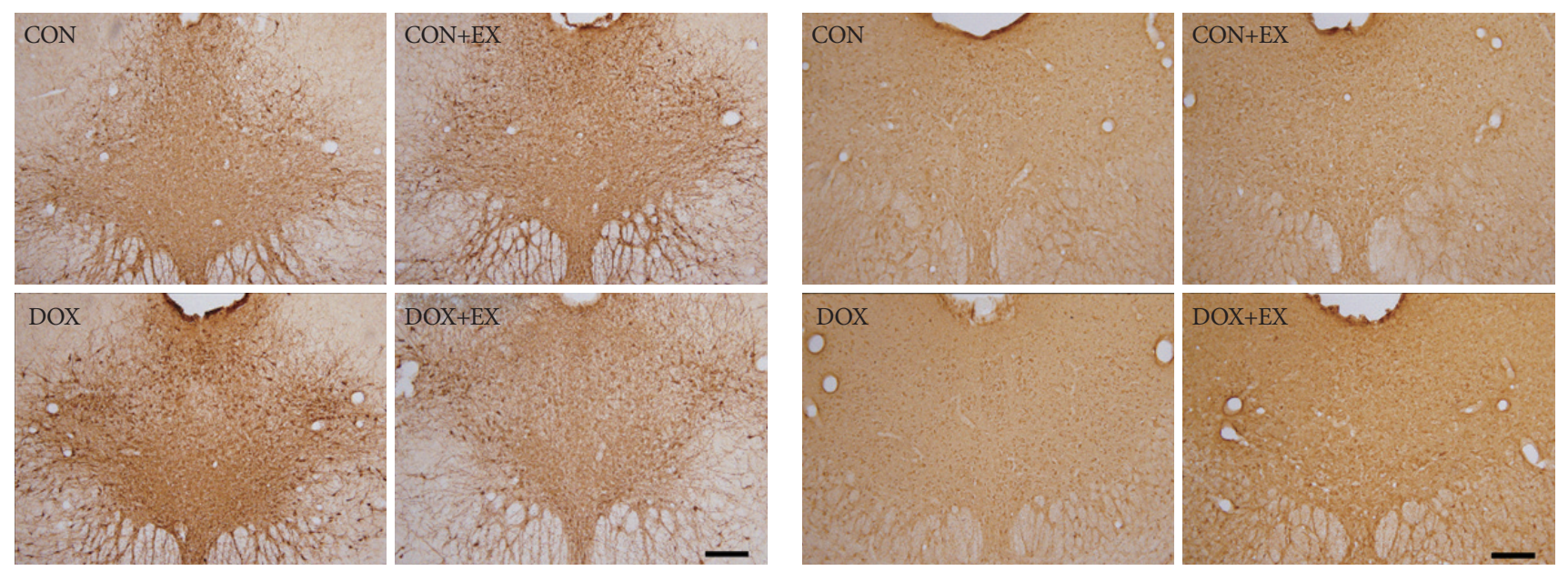

A
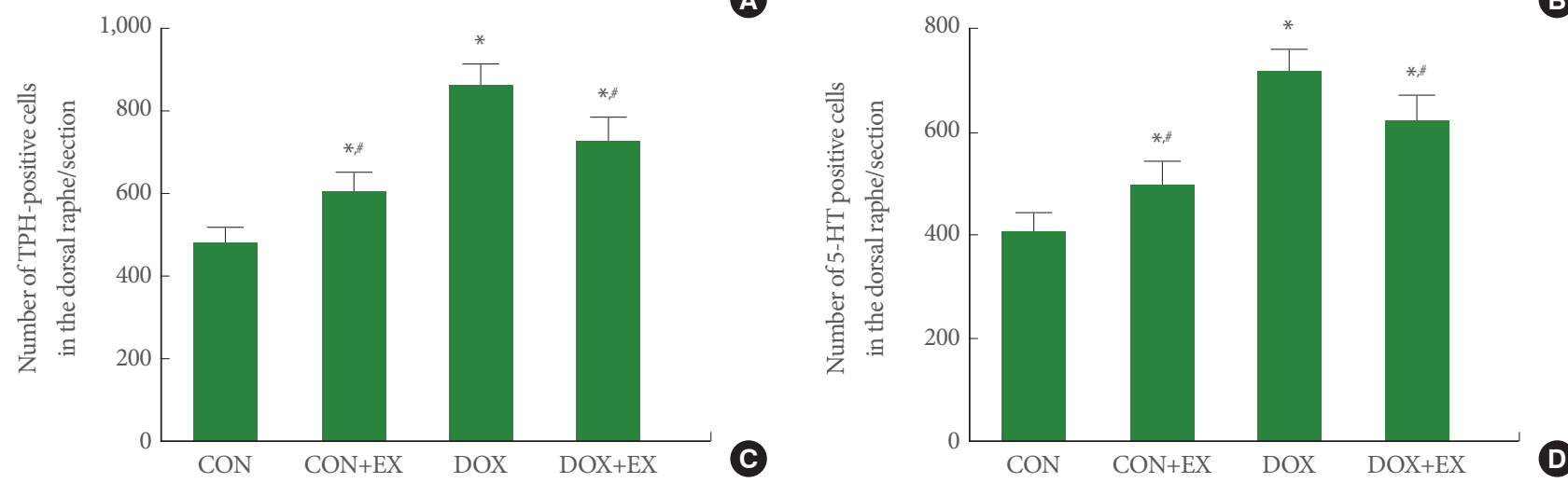

Fig. 2. The effect of treadmill exercise on expressions of tryptophan hydroxylase (TPH) and 5-hydroxytryptamine (5-HT)-positive cells in the dorsal raphe. (A) Photomicrographs of TPH. (B) The number of TPH-positive cells in each group. (C) Photomicrographs of 5-HT. (D) The number of 5-HT-positive cells in each group. The scale bar represents $200 \mu \mathrm{m}$. CON, control group; CON+EX, control with exercise group; DOX, doxorubicin (DOX)-treated group; DOX+EX, DOX-treated with exercise group. Data are expressed as the mean \pm standard error of the mean. ${ }^{*} \mathrm{P}<0.05$ compared to control group. ${ }^{*} \mathrm{P}<0.05$ compared to DOX-treated group.

expression was increase by DOX treatment $(\mathrm{P}<0.05)$, in contrast, treadmill exercise decreased caspases- 3 expression in the DOX-treated group $(\mathrm{P}<0.05)$.

\section{Mitochondrial $\mathrm{O}_{2}$ Respiration in Gastrocnemius}

Fig. $4 \mathrm{~A}$ shows the results of mitochondrial $\mathrm{O}_{2}$ respiration in the gastrocnemius. Mitochondrial $\mathrm{O}_{2}$ respiration under the stage of each factor (glutamate and malate infusion; adenosine diphosphate infusion; succinate infusion) was decreased by DOX treatment $(\mathrm{P}<0.05)$. Meanwhile, treadmill exercise increased mitochondrial $\mathrm{O}_{2}$ respiration under the stage of each factor in the DOX-treated group $(\mathrm{P}<0.05)$.

\section{Mitochondrial $\mathrm{H}_{2} \mathrm{O}_{2}$ Emission in Gastrocnemius}

Fig. $4 \mathrm{~B}$ shows the results of mitochondrial $\mathrm{H}_{2} \mathrm{O}_{2}$ emission in the gastrocnemius. Mitochondrial $\mathrm{H}_{2} \mathrm{O}_{2}$ emission under the stage of each factor (glutamate and malate infusion; glutamate, malate, and succinate infusion; glutamate, malate, succinate, and glycerol 3-phosphate infusion) was increased by DOX treatment $(\mathrm{P}<0.05)$. Meanwhile, treadmill exercise decreased mitochondrial $\mathrm{H}_{2} \mathrm{O}_{2}$ emission under the stage of each factor in the DOX-treated group $(\mathrm{P}<0.05)$.

\section{Mitochondrial $\mathrm{Ca}^{2+}$ Retention Capacity in the Gastrocnemius}

Fig. $4 \mathrm{C}$ shows the results of mitochondrial $\mathrm{Ca}^{2+}$ retention capacity in the gastrocnemius. Mitochondrial $\mathrm{Ca}^{2+}$ retention capacity was decreased by DOX treatment $(\mathrm{P}<0.05)$. Meanwhile, treadmill exercise increased mitochondrial $\mathrm{Ca}^{2+}$ retention capacity in the DOX-treated group $(\mathrm{P}<0.05)$. 

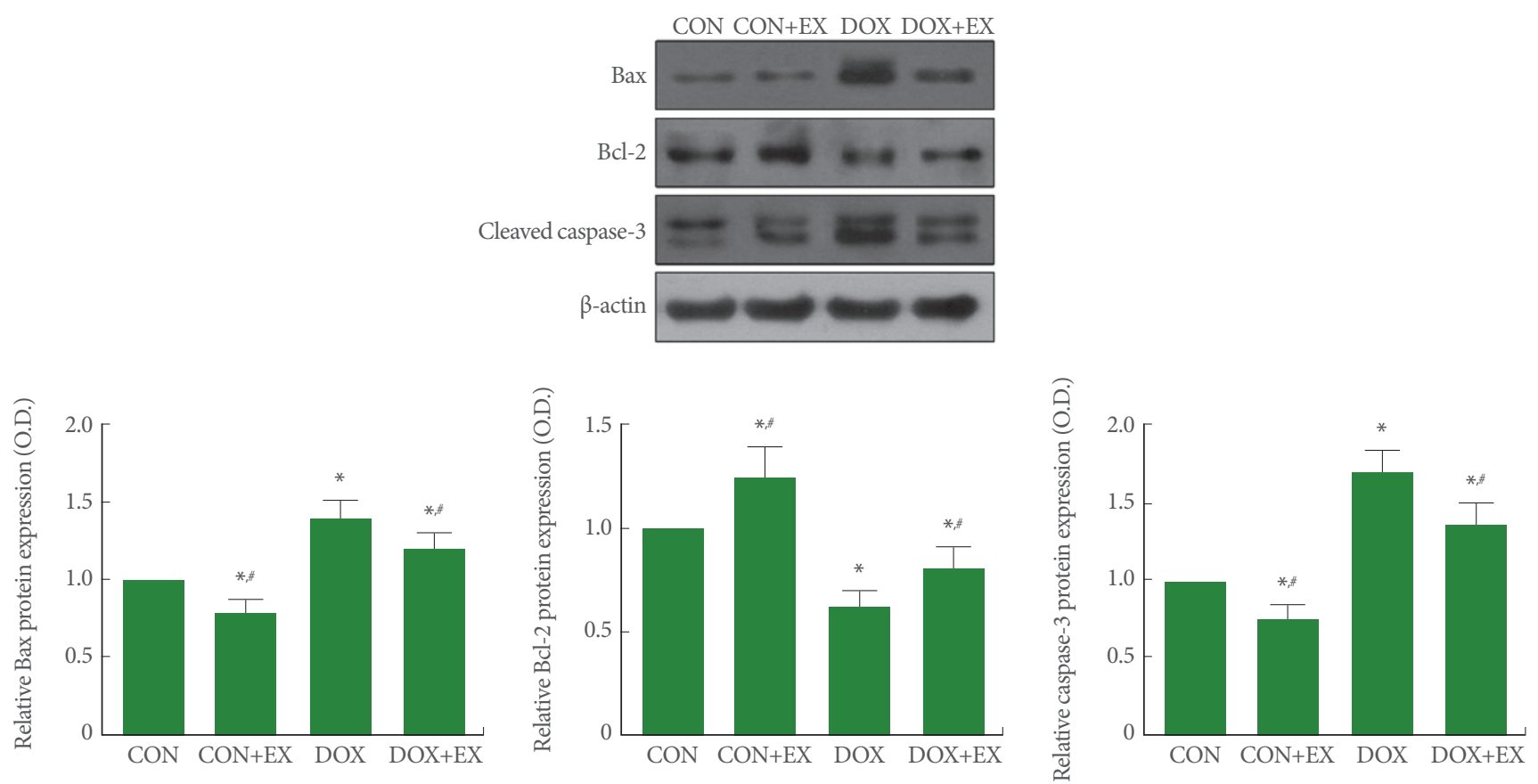

Fig. 3. The effect of treadmill exercise on apoptosis in the gastrocnemius. Upper panel: Representative Bax, Bcl-2, and caspase- 3 expressions. Lower panel: Relative optical density of Bax, Bcl-2, and caspase-3 in each group. CON, control group; CON+EX, control with exercise group; DOX, doxorubicin (DOX)-treated group; DOX+EX, DOX-treated with exercise group. Data are expressed as the mean \pm standard error of the mean. ${ }^{*} \mathrm{P}<0.05$ compared to control group. $\# \mathrm{P}<0.05$ compared to DOX-treated group.
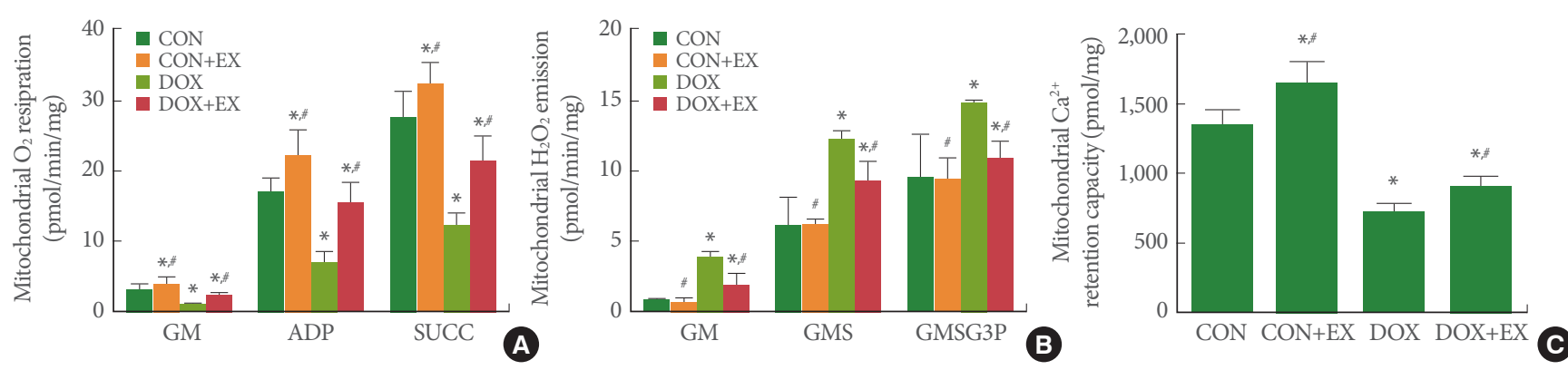

Fig. 4. The effect of treadmill exercise on mitochondrial function in the gastrocnemius. (A) Mitochondrial $\mathrm{O}_{2}$ respiration. (B) Mitochondrial $\mathrm{H}_{2} \mathrm{O}_{2}$ emission. (C) Mitochondrial $\mathrm{Ca}^{2+}$ retention capacity. GM, glutamate and malate infusion; ADP, adenosine diphosphate infusion; SUCC, succinate infusion; GMS, glutamate, malate, and succinate infusion; GMS3P, glutamate, malate, succinate, and glycerol 3-phosphate infusion. CON, control group; CON+EX, control with exercise group; DOX, doxorubicin (DOX)-treated group; $\mathrm{DOX}+\mathrm{EX}, \mathrm{DOX}$-treated with exercise group. Data are expressed as the mean \pm standard error of the mean. ${ }^{*} \mathrm{P}<0.05$ compared to control group. ${ }^{\#} \mathrm{P}<0.05$ compared to DOX-treated group.

\section{DISCUSSION}

Anticancer drugs are absolutely necessary treatment for cancer patient, however, the use of these anticancer drugs including DOX is limited by their toxicity. DOX causes side effects such as generation of ROS, stimulation of p53-DNA binding, activation of caspases, DNA cross-linking, and DNA damage [1-3,31].
In this study, enhanced Bax expression and suppressed Bcl-2 expression appeared in the gastrocnemius by DOX treatment, demonstrating that DOX facilitates apoptosis in the muscle. DOX diminishes antioxidant capacity and promotes apoptosis through increasing oxidative stress, and then induces mitochondrial DNA damage $[9,11,14]$. In this result, Bax expression was suppressed and $\mathrm{Bcl}-2$ expression in the gastrocnemius was 
enhanced by treadmill exercise, demonstrating that treadmill exercise inhibited DOX-caused apoptotic cell death in the muscle. Suppressing effect of treadmill running on apoptosis is well documented [30,32].

In the present study, $\mathrm{O}_{2}$ respiration and $\mathrm{Ca}^{2+}$ retention capacity in the mitochondria of gastrocnemius were decreased and $\mathrm{H}_{2} \mathrm{O}_{2}$ emission in the mitochondria of gastrocnemius was increased by DOX treatment. PTP sensitivity of mitochondria is influenced by ROS production and $\mathrm{Ca}^{2+}$ homeostasis, and mitochondrial function is diminished when apoptosis is exacerbated $[14,17]$. In the present result, treadmill exercise enhanced $\mathrm{O}_{2}$ respiration and $\mathrm{Ca}^{2+}$ retention capacity in the mitochondria of gastrocnemius and suppressed $\mathrm{H}_{2} \mathrm{O}_{2}$ emission in the mitochondria of gastrocnemius, demonstrating that treadmill exercise is effective for the improving mitochondrial function of muscle. Exercise is known to increase mitochondrial plasticity and $\mathrm{Ca}^{2+}$ retention, as a result, exercise alleviates the degree of oxidative stress $[9,13]$.

In the present study, DOX treatment reduced grip strength and increased fatigue index of the gastrocnemius. Increased oxidative stress in the skeletal muscle weakens muscle strength and then causes muscle fatigue $[18,19]$. In the present result, treadmill exercise increased grip strength and reduced fatigue index, demonstrating that treadmill exercise improves muscle fatigue by reducing DOX-induced muscle weakness. It is known that exercise during chemotherapy can reduce muscle damage caused by DOX [20].

Muscle weakness and muscle fatigue are the common side effects during DOX therapy. Over-expression of TPH and 5-HT in the dorsal raphe is known to be an important factor for central fatigue induction [22,23]. In this study, it was observed that the expressions of TPH and 5-HT in the dorsal raphe were suddenly increased by DOX treatment, suggesting that that DOX therapy induces central fatigue as well as muscle fatigue. 5-HT synthesis and TPH expression in the dorsal raphe are well documented to be enhanced by treadmill exercise $[29,33]$. In present result, however, treadmill exercise suppressed DOX-induced over-expression of TPH and 5-HT in the dorsal raphe. This suppression on 5-HT synthesis and TPH expression by treadmill running might contribute to the amelioration of DOX-induced central fatigue.

In conclusion, DOX increased apoptosis and decreased mitochondria function in muscle, resulting in muscle weakness and central fatigue. Treadmill exercise suppressed apoptosis and prevented deterioration of mitochondria function in mus- cle, resulting in alleviation of muscle weakness and central fatigue during DOX therapy.

\section{AUTHOR CONTRIBUTION STATEMENT}

- Full access to all the data in the study and takes responsibility for the integrity of the data and the accuracy of the data analysis: SSP

- Study concept and design: TWK

- Acquisition of data: HSP, MHN, JWH, SZY

- Drafting of the manuscript: SSP

- Critical revision of the manuscript for important intellectual content: HSP, MHN, JWH, SZY

- Obtained funding: TWK

- Administrative, technical, or material support: $H B K$

-Study supervision: TWK, HBK

\section{REFERENCES}

1. Serrano J, Palmeira CM, Kuehl DW, Wallace KB. Cardioselective and cumulative oxidation of mitochondrial DNA following subchronic doxorubicin administration1. Biochim Biophys Acta 1999; 1411:201-5.

2. Gilliam LA, Moylan JS, Callahan LA, Sumandea MP, Reid MB. Doxorubicin causes diaphragm weakness in murine models of cancer chemotherapy. Muscle Nerve 2011;43:94-102.

3. Gilliam LA, Moylan JS, Ferreira LF, Reid MB. TNF/TNFR1 signaling mediates doxorubicin-induced diaphragm weakness. Am J Physiol Lung Cell Mol Physiol 2011;300:L225-31.

4. Vichaya EG, Chiu GS, Krukowski K, Lacourt TE, Kavelaars A, Dantzer R, et al. Mechanisms of chemotherapy-induced behavioral toxicities. Front Neurosci 2015;9:131.

5. Zhou S, Starkov A, Froberg MK, Leino RL, Wallace KB. Cumulative and irreversible cardiac mitochondrial dysfunction induced by doxorubicin. Cancer Res 2001;61:771-7.

6. Oliveira PJ, Bjork JA, Santos MS, Leino RL, Froberg MK, Moreno $\mathrm{AJ}$, et al. Carvedilol-mediated antioxidant protection against doxorubicin-induced cardiac mitochondrial toxicity. Toxicol Appl Pharmacol 2004;200:159-68.

7. van Norren K, van Helvoort A, Argilés JM, van Tuijl S, Arts K, Gorselink, $\mathrm{M}$ et al. Direct effects of doxorubicin on skeletal muscle contribute to fatigue. Br J Cancer 2009;100:311-4.

8. Yamamoto Y, Hoshino Y, Ito T, Nariai T, Mohri T, Obana M, et al. Atrogin-1 ubiquitin ligase is upregulated by doxorubicin via p38MAP kinase in cardiac myocytes. Cardiovasc Res 2008;79:89-96. 
9. Yu AP, Pei XM, Sin TK, Yip SP, Yung BY, Chan LW, et al. Acylated and unacylated ghrelin inhibit doxorubicin-induced apoptosis in skeletal muscle. Acta Physiol (Oxf) 2014;211:201-13.

10. António A, Magalhães J, Soares J, Ferreira R, Neuparth M, Marques $\mathrm{F}$, et al. Endurance exercise training attenuates morphological signs of cardiac muscle damage induced by doxorubicin in male mice. Basic Appl Myol 2006;16:27-35.

11. Green PS, Leeuwenburgh C. Mitochondrial dysfunction is an early indicator of doxorubicin-induced apoptosis. Biochim Biophys Acta 2002;1588:94-101.

12. Childs AC, Phaneuf SL, Dirks AJ, Phillips T, Leeuwenburgh C. Doxorubicin treatment in vivo causes cytochrome $\mathrm{C}$ release and cardiomyocyte apoptosis, as well as increased mitochondrial efficiency, superoxide dismutase activity, and Bcl-2:Bax ratio. Cancer Res 2002;62:4592-8.

13. Kim SY, Kim SJ, Kim BJ, Rah SY, Chung SM, Im MJ, et al. Doxorubicin-induced reactive oxygen species generation and intracellular $\mathrm{Ca}^{2+}$ increase are reciprocally modulated in rat cardiomyocytes. Exp Mol Med 2006;38:535-45.

14. Tangpong J, Cole MP, Sultana R, Joshi G, Estus S, Vore M, et al. Adriamycin-induced, TNF-alpha-mediated central nervous system toxicity. Neurobiol Dis 2006;23:127-39.

15. Tangpong J, Cole MP, Sultana R, Estus S, Vore M, St Clair W, et al. Adriamycin-mediated nitration of manganese superoxide dismutase in the central nervous system: insight into the mechanism of chemobrain. J Neurochem 2007;100:191-201.

16. Smuder AJ, Kavazis AN, Min K, Powers SK. Exercise protects against doxorubicin-induced oxidative stress and proteolysis in skeletal muscle. J Appl Physiol (1985) 2011;110:935-42.

17. Supinski GS, Callahan LA. Free radical-mediated skeletal muscle dysfunction in inflammatory conditions. J Appl Physiol (1985) 2007;102:2056-63.

18. Powers SK, Jackson MJ. Exercise-induced oxidative stress: cellular mechanisms and impact on muscle force production. Physiol Rev 2008;88:1243-76.

19. Kavazis AN, Smuder AJ, Powers SK. Effects of short-term endurance exercise training on acute doxorubicin-induced FoxO transcription in cardiac and skeletal muscle. J Appl Physiol (1985) 2014;117:223-30.

20. Smuder AJ, Kavazis AN, Min K, Powers SK. Exercise protects against doxorubicin-induced markers of autophagy signaling in skeletal muscle. J Appl Physiol (1985) 2011;111:1190-8.

21. Ascensão A, Lumini-Oliveira J, Machado NG, Ferreira RM, Gon- çalves IO, Moreira AC, et al. Acute exercise protects against calcium-induced cardiac mitochondrial permeability transition pore opening in doxorubicin-treated rats. Clin Sci (Lond) 2011;120:3749.

22. Davis JM, Alderson NL, Welsh RS. Serotonin and central nervous system fatigue: nutritional considerations. Am J Clin Nutr 2000; 72:573S-578S.

23. Seo JH, Sung YH, Kim KJ, Shin MS, Lee EK, Kim CJ. Effects of Phellinus linteus administration on serotonin synthesis in the brain and expression of monocarboxylate transporters in the muscle during exhaustive exercise in rats. J Nutr Sci Vitaminol (Tokyo). 2011;57:95-103.

24. Blomstrand E. Amino acids and central fatigue. Amino Acids 2001;20:25-34.

25. Blomstrand E. A role for branched-chain amino acids in reducing central fatigue. J Nutr 2006;136:544S-547S.

26. Fernstrom JD, Fernstrom MH. Exercise, serum free tryptophan, and central fatigue. J Nutr 2006;136:553S-559S.

27. Foley TE, Fleshner M. Neuroplasticity of dopamine circuits after exercise: implications for central fatigue. Neuromolecular Med 2008;10:67-80.

28. Park HS, Kim CJ, Kwak HB, No MH, Heo JW, Kim TW. Physical exercise prevents cognitive impairment by enhancing hippocampal neuroplasticity and mitochondrial function in doxorubicin-induced chemobrain. Neuropharmacology 2018;133:451-61.

29. Ji ES, Lee JM, Kim TW, Kim YM, Kim YS, Kim K. Treadmill exercise ameliorates depressive symptoms through increasing serotonin expression in postpartum depression rats. J Exerc Rehabil 2017; 13:130-5.

30. Song SH, Jee YS, Ko IG, Lee SW, Sim YJ, Kim DY, et al. Treadmill exercise and wheel exercise improve motor function by suppressing apoptotic neuronal cell death in brain inflammation rats. J Exerc Rehabil 2018;27;14:911-9.

31. Tsujimoto Y1, Shimizu S. Role of the mitochondrial membrane permeability transition in cell death. Apoptosis 2007;12:835-40.

32. Lee JM, Ji ES, Kim TW, Kim CJ, Shin MS, Lim BV, et al. Treadmill exercise improves memory function by inhibiting hippocampal apoptosis in pilocarpine-induced epileptic rats. J Exerc Rehabil 2018;14:713-23.

33. Cho JW, Jung SY, Lee SW, Lee SJ, Seo TB, Kim YP, et al. Treadmill exercise ameliorates social isolation-induced depression through neuronal generation in rat pups. J Exerc Rehabil 2017;13:627-33. 